EPJ Web of Conferences 97,00015 (2015)

DOI: 10.1051/epjconf/201597000 15

(C) Owned by the authors, published by EDP Sciences, 2015

\title{
Highlights of Resonance Measurements With HADES
}

Eliane Epple ${ }^{10,9, a}$, for the HADES collaboration:

J. Adamczewski-Musch ${ }^{4}$, O. Arnold ${ }^{10,9}$, E.T. Atomssa ${ }^{15}$, C. Behnke ${ }^{8}$, J.C. Berger-Chen ${ }^{10,9}$, J. Biernat ${ }^{3}$, A. Blanco ${ }^{2}$, C. Blume ${ }^{8}$, M. Böhmer ${ }^{10}$, P. Bordalo ${ }^{2}$, S. Chernenko ${ }^{7}$, C. Deveaux ${ }^{11}$, A. Dybczak ${ }^{3}$, L. Fabbietti ${ }^{10,9}$, O. Fateev ${ }^{7}$, P. Fonte ${ }^{2,18}$, C. Franco ${ }^{2}$, J. Friese ${ }^{10}$, I. Fröhlich ${ }^{8}$, T. Galatyuk ${ }^{5,19}$, J. A. Garzón ${ }^{17}$, K. Gill ${ }^{8}$, M. Golubeva ${ }^{12}$, F. Guber ${ }^{12}$, M. Gumberidze ${ }^{5,19}$, S. Harabasz ${ }^{5,3}$, T. Hennino ${ }^{15}$, S. Hlavac ${ }^{1}$, C. Höhne ${ }^{11}$, R. Holzmann ${ }^{4}$, A. lerusalimov ${ }^{7}$, A. Ivashkin ${ }^{12}$, M. Jurkovic ${ }^{10}$, B. Kämpfer ${ }^{6,20}$, T. Karavicheva ${ }^{12}$, K. Kardan ${ }^{8}$, I. Koenig ${ }^{4}$, W. Koenig ${ }^{4}$, B. W. Kolb ${ }^{4}$, G. Korcyl ${ }^{3}$, G. Kornakov ${ }^{5}$, R. Kotte ${ }^{6}$, A. Krása ${ }^{16}$, E. Krebs ${ }^{8}$, H. Kuc ${ }^{3,15}$, A. Kugler ${ }^{16}$, T. Kunz ${ }^{10}$, A. Kurepin ${ }^{12}$, A. Kurilkin ${ }^{7}$, P. Kurilkin ${ }^{7}$, V. Ladygin ${ }^{7}$, R. Lalik ${ }^{10,9}$, K. Lapidus ${ }^{10,9}$, A. Lebedev ${ }^{13}$, L. Lopes ${ }^{2}$, M. Lorenz ${ }^{8}$, T. Mahmoud $^{11}$, L. Maier ${ }^{10}$, A. Mangiarotti ${ }^{2}$, J. Markert $^{8}$, V. Metag ${ }^{11}$, J. Michel ${ }^{8}$, C. Müntz ${ }^{8}$, R. Münzer ${ }^{10,9}$, L. Naumann ${ }^{6}$, M. Palka ${ }^{3}$, Y. Parpottas ${ }^{14,21}$, V. Pechenov ${ }^{4}$, O. Pechenova ${ }^{8}$, V. Petousis ${ }^{14}$, J. Pietraszko ${ }^{4}$, W. Przygoda ${ }^{3}$, B. Ramstein ${ }^{15}$, L. Rehnisch ${ }^{8}$, A. Reshetin ${ }^{12}$, A. Rost ${ }^{5}$, A. Rustamov 8 , A. Sadovsky ${ }^{12}$, P. Salabura ${ }^{3}$, T. Scheib ${ }^{8}$, K. Schmidt-Sommerfeld ${ }^{10}$, H. Schuldes ${ }^{8}$, P. Sellheim ${ }^{8}$, J. Siebenson ${ }^{10}$, L. Silva ${ }^{2}$, Yu.G. Sobolev ${ }^{16}$, S. Spataro ${ }^{22}$, H. Ströbele ${ }^{8}$, J. Stroth ${ }^{8,4}$, P. Strzempek ${ }^{3}$, C. Sturm ${ }^{4}$, O. Svoboda ${ }^{16}$, A. Tarantola ${ }^{8}$, K. Teilab ${ }^{8}$, P. Tlusty ${ }^{16}$, M. Traxler ${ }^{4}$, H. Tsertos ${ }^{14}$, T. Vasiliev ${ }^{7}$, V. Wagner ${ }^{16}$, C. Wendisch ${ }^{6,20}$, J. Wirth ${ }^{10,9}$, J. Wüstenfeld ${ }^{6}$, Y. Zanevsky ${ }^{7}$, and P. Zumbruch ${ }^{4}$

${ }^{1}$ Institute of Physics, Slovak Academy of Sciences, 84228 Bratislava, Slovakia

${ }^{2}$ LIP-Laboratório de Instrumentação e Física Experimental de Partículas , 3004-516 Coimbra, Portugal

${ }^{3}$ Smoluchowski Institute of Physics, Jagiellonian University of Cracow, 30-059 Kraków, Poland

${ }^{4}$ GSI Helmholtzzentrum für Schwerionenforschung GmbH, 64291 Darmstadt, Germany

${ }^{5}$ Technische Universität Darmstadt, 64289 Darmstadt, Germany

${ }^{6}$ Institut für Strahlenphysik, Helmholtz-Zentrum Dresden-Rossendorf, 01314 Dresden, Germany

${ }^{7}$ Joint Institute of Nuclear Research, 141980 Dubna, Russia

${ }^{8}$ Institut für Kernphysik, Goethe-Universität, 60438 Frankfurt, Germany

${ }^{9}$ Excellence Cluster 'Origin and Structure of the Universe', 85748 Garching, Germany

${ }^{10}$ Physik Department E12, Technische Universität München, 85748 Garching, Germany

${ }^{11}$ II.Physikalisches Institut, Justus Liebig Universität Giessen, 35392 Giessen, Germany

${ }^{12}$ Institute for Nuclear Research, Russian Academy of Science, 117312 Moscow, Russia

${ }^{13}$ Institute of Theoretical and Experimental Physics, 117218 Moscow, Russia

${ }^{14}$ Department of Physics, University of Cyprus, 1678 Nicosia, Cyprus

${ }^{15}$ Institut de Physique Nucléaire (UMR 8608), CNRS/IN2P3 - Université Paris Sud, F-91406 Orsay Cedex, France

${ }^{16}$ Nuclear Physics Institute, Academy of Sciences of Czech Republic, 25068 Rez, Czech Republic

${ }^{17}$ LabCAF. F. Física, Univ. de Santiago de Compostela, 15706 Santiago de Compostela, Spain

${ }^{18}$ also at ISEC Coimbra, Coimbra, Portugal

${ }^{19}$ also at ExtreMe Matter Institute EMMI, 64291 Darmstadt, Germany

${ }^{20}$ also at Technische Universität Dresden, 01062 Dresden, Germany

${ }^{21}$ also at Frederick University, 1036 Nicosia, Cyprus

\footnotetext{
ae-mail: eliane.epple@ph.tum.de
}

This is an Open Access article distributed under the terms of the Creative Commons Attribution License 4.0, which permits unrestricted use, distribution, and reproduction in any medium, provided the original work is properly cited. 


\begin{abstract}
This contribution aims to give a basic overview of the latest results regarding the production of resonances in different collision systems. The results were extracted from experimental data collected with HADES that is a multipurpose detector located at the GSI Helmholtzzentrum, Darmstadt. The main points discussed here are: the properties of the strange resonances $\Lambda(1405)$ and $\Sigma(1385)$, the role of $\Delta$ 's as a source of pions in the final state, the production dynamics reflected in form of differential cross sections, and the role of the $\phi$ meson as a source for $\mathrm{K}^{-}$particles.
\end{abstract}

\title{
1 Introduction
}

In the last years new efforts have been set to gain a comprehensive understanding for resonance production in elementary, and consequently also heavy ion reactions. One example is the propagation of particles with a high absorption probability through nuclear matter. Anti-kaons, for instance, strongly couple to strange resonances in the nuclear medium via reactions, like:

$$
\begin{aligned}
& \bar{K} N \rightarrow \Lambda(1405) \rightarrow \bar{K} N / \Sigma \pi, \\
& \bar{K} N \rightarrow \Sigma(1385) \rightarrow \bar{K} N / \Sigma \pi / \Lambda \pi .
\end{aligned}
$$

To understand the production kinematics and in-medium propagation of anti-kaons, the basic properties of these resonances have to be correctly taken into account in transport models, for instance.

Other observables, like inverse slope parameters of transverse momentum spectra, reflect directly the influence of resonances. A nice example is the extraction of different effective temperatures ${ }^{1}$ for $\mathrm{K}^{+}$and $\mathrm{K}^{-}$in $\mathrm{Ar}+\mathrm{KCl}$ collisions [1]. This observation is, in fact, not a sign of a different thermal decoupling of the two mesons from the medium but originates from the different production mechanisms for the two kaon species [2]. Namely, a large fraction of $\mathrm{K}^{-}$stems from the $\phi$ decay which modifies their observed spectrum.

Starting from these and other observations, HADES has intensively studied resonance production in several light colliding systems $(\mathrm{p}+\mathrm{p}, \mathrm{d}+\mathrm{p}, \mathrm{p}+\mathrm{Nb})$ to build a solid basis for the understanding of hadron properties in heavier collision systems. Of large interest in these investigations were the properties of the first excited strange resonances $\Lambda(1405)$ and $\Sigma(1385)[3,4]$. Further, the role of $\Delta$ and $\mathrm{N}^{*}$ production was investigated in several works in elementary reactions at different beam kinetic energies [5-8]. An objective was the extraction of the individual contributions of the resonances to the total production cross section, as well as their production and decay properties which are encoded in angular distributions in different reference frames. An extension of the studies regarding the $\phi / K^{-}$ ratio in $\mathrm{Ar}+\mathrm{KCl}$ reactions is the reconstruction of this quantity in the $\mathrm{Au}+\mathrm{Au}(1.23 \mathrm{GeV} / \mathrm{A})$ collision system.

The data, presented here, were all collected with the HADES experiment which is a versatile detector installed as a fixed target experiment at the GSI Helmholtzzentrum. For details about the experimental setup see Ref. [9].

\section{Strange Resonances by HADES}

The importance of resonance properties for the in-medium anti-kaon propagation was the motivation for two analyses regarding the strange resonances $\Lambda(1405)$ and $\Sigma(1385)^{+}$with HADES. Both resonances were reconstructed in $\mathrm{p}+\mathrm{p}$ collisions at a beam kinetic energy of $3.5 \mathrm{GeV}$. While the $\Sigma(1385)^{+}$

\footnotetext{
${ }^{1}$ This corresponds to the inverse slope parameter of the transverse momentum distribution at mid-rapidity
} 


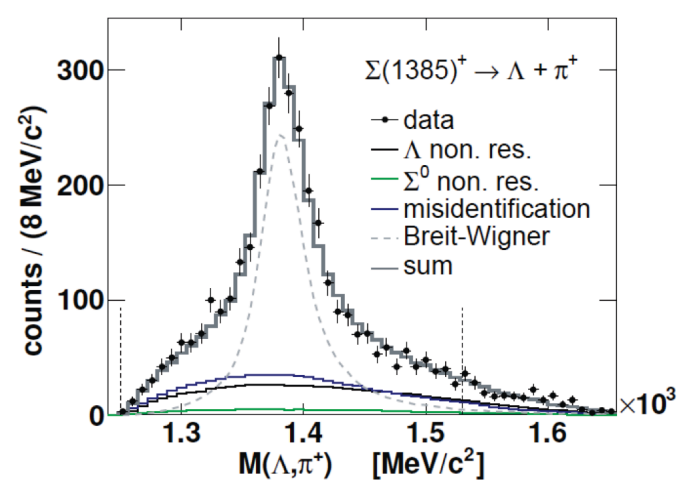

Figure 1. (Color online) The $\Lambda \pi^{+}$invariant mass distribution from Reaction (3) [3]. The contribution from the $\Sigma(1385)^{+}$resonance as well as background channels is shown by the different colored histograms.

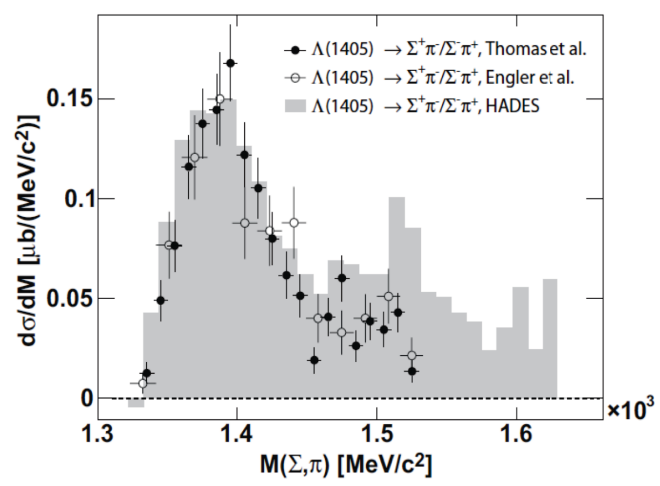

Figure 2. The line shape of the $\Lambda(1405)$ reconstructed from Reaction 4 is compared to measurements with pion induced reactions [13] of Thomas et al. [14] and Engler et al. [15].

is understood as a three quark resonance, the $\Lambda(1405)$ is treated as a bound meson-baryon system consisting of two interfering poles in most of the theories [10]. This property makes the measured line shape of the $\Lambda(1405)$ a particularly interesting observable, as it is believed that the entrance channel in which the $\Lambda(1405)$ is produced can influence the appearance of its mass spectrum due to its peculiar sub-structure.

The $\Sigma(1385)^{+}$was reconstructed in the reaction:

$$
p+p \rightarrow \Sigma(1385)^{+} n K^{+},
$$

where the $\Lambda \pi^{+}$decay of the resonance was exploited to reconstruct the complete event kinematic [3]. The reconstructed Breit-Wigner distribution is illustrated in Figure 1. The extracted values for the mass $m_{0}=1383.2 \pm 0.9_{-1.5}^{+0.1} \mathrm{MeV} / \mathrm{c}^{2}$ and $\Gamma_{0}=40.2 \pm 2.1_{-2.8}^{+1.2} \mathrm{MeV} / \mathrm{c}^{2}$, agree well within the uncertainties, with the literature value [11]. 
For the reconstruction of the $\Lambda(1405)$ resonance the following reaction was considered [4]

$$
p+p \rightarrow \Lambda(1405) p K^{+} \rightarrow\left(\Sigma^{\mp} \pi^{ \pm}\right) p K^{+} .
$$

The acceptance and efficiency corrected mass distribution of the $\Lambda$ (1405) (including background contributions) is shown in Figure 2. As the line shape of the resonance is the most important observable and is expected to change in different production reactions, we have compared it to the mass distributions measured in pion induced reactions. In contrast to e.g. gamma induced reactions [12], the three line shapes in Figure 2 agree well with one another [13]. This might hint towards a similar production mechanism in pion and proton induced reactions. The maximum of the mass distribution differs from kaon induced reactions [4] and this feature should be implemented carefully in models that treat the in-medium behavior of anti-kaons.
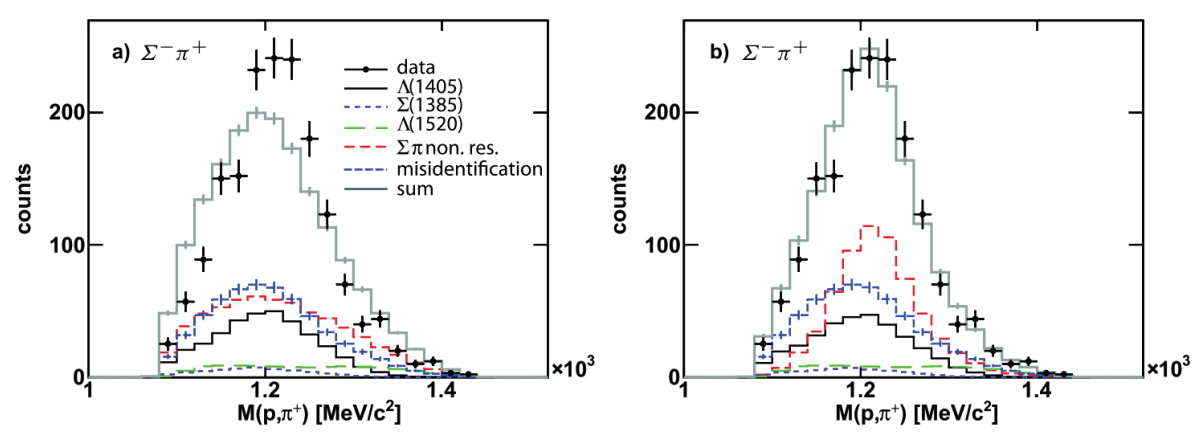

Figure 3. $\pi^{+} p$ invariant mass spectra from the analysis of the $\Lambda(1405)$ resonance [4]. The left side shows the data compared to simulation where the $\pi^{+} p$ from Reaction (5) are produced directly, while the right side shows the data compared to simulation where the $\pi^{+} p$ from Reaction (5) stem from the decay of the $\Delta^{++}$resonance (both shown as red histogram). In the second case, the data are much better described by the sum of all contributions (gray histogram) [16].

\section{The Importance of $\Delta$ 's}

The investigation of the $\Lambda(1405)$ resonance has yielded, apart from the first high quality result of the resonance line shape in $\mathrm{p}+\mathrm{p}$ collisions, another interesting outcome. Namely, the nature of the background underneath the resonance signal [16]. The two components, discussed in the following are:

$$
\begin{aligned}
& p+p \rightarrow \Sigma^{-} \pi^{+} p K^{+}, \\
& p+p \rightarrow \Sigma^{+} \pi^{-} p K^{+} .
\end{aligned}
$$

The investigation of the invariant mass distribution of $\pi^{+} p$ in Reaction (5) has shown, that the data are best described by assuming that these two particles stem from the decay of a $\Delta^{++}(1232)$ resonance, while Reaction (6) gave no hint, that the $\pi^{-} p$ pair might stem from a $\Delta^{0}(1232)$ resonance [16]. Figure 3 illustrates the former case. The left panel shows that if the simulation of Reaction (5) is included as a non-resonant contribution, the data are not well described by the cocktail of all contributing sources. 

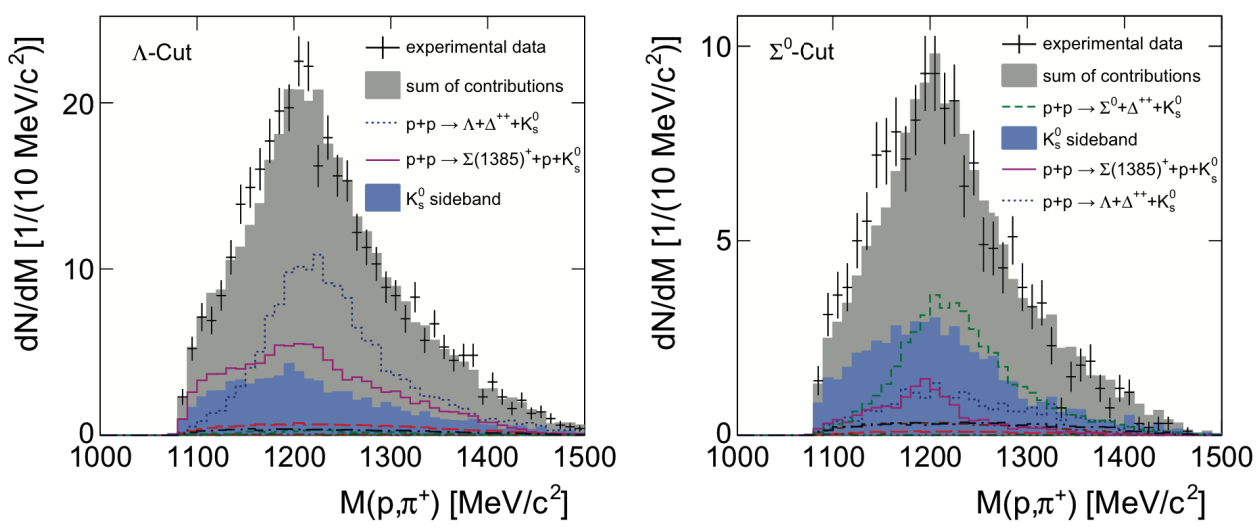

Figure 4. $\pi^{+} p$ invariant mass spectra from the analysis of the associate production of $K^{0}$ mesons [5]. The left panel shows events that contain a $\Lambda$, while the right panel shows events with a $\Sigma^{0}$. The gray histograms correspond to the sum of simulated contributions plus the non-strange background described by the $K^{0}$ side band sample. Detailed information can be found in Ref. [5].

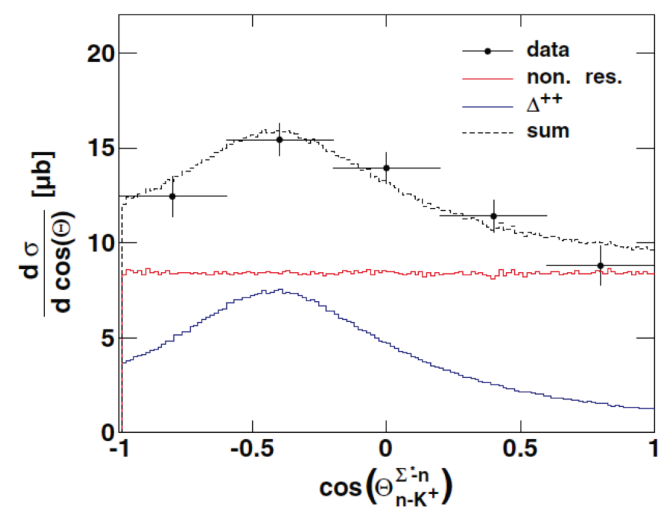

Figure 5. Angular differential cross section of Reaction (3) as a function of the cosine of the angle between the $\Sigma^{*}$ and the neutron in the $n-K^{+}$-rest-frame (helicity angle). The observable is described best by the simulation, if about $33 \%$ of the $\Sigma^{*}$ are produced via a higher mass resonance (blue histogram) [3].

If, however, the simulations are modified to include the $\Delta^{++}(1232)$ as the source of the $\pi^{+} p$ pair in Reaction (5), the simulation describe the data much better [16]. Hence, we conclude that a $\Sigma^{-}$and a $K^{+}$in $\mathrm{p}+\mathrm{p}$ collisions are predominantly accompanied by a $\Delta^{++}(1232)$, if an additional pion is in the final state.

Complementary to the analysis of resonances produced with $K^{+}$mesons, an analysis of exclusive production channels including $K^{0}$ has recently been presented [5]. In this analysis the reactions

$$
\begin{gathered}
p+p \rightarrow \Lambda \pi^{+} p K^{0}, \\
p+p \rightarrow \Sigma^{0} \pi^{+} p K^{0},
\end{gathered}
$$



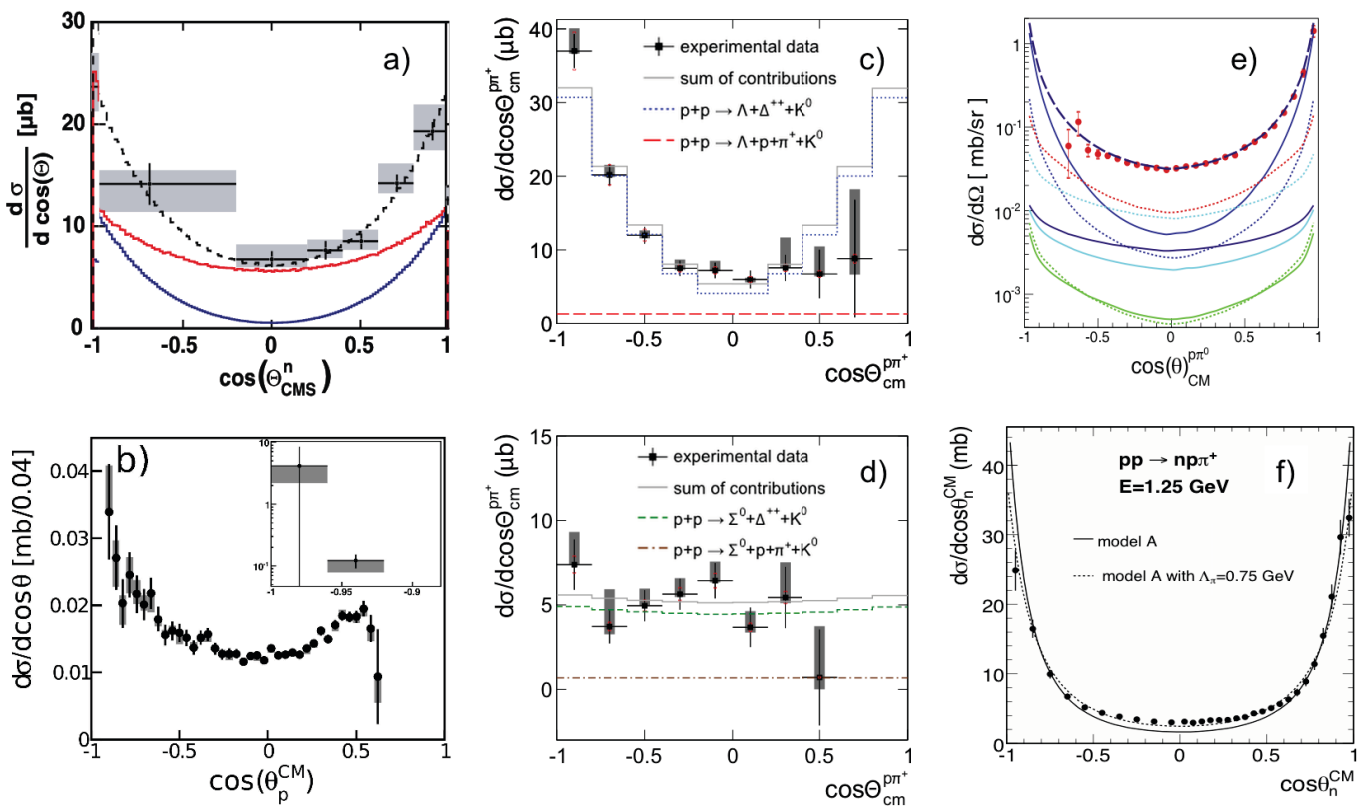

Figure 6. This figure is a compilation of many reconstructed angular distributions of different data measured with the HADES spectrometer. Panel a) shows the neutron CMS angle of Reaction (3) [3]. Panel b) shows the proton CMS angle from the exclusive $p K^{+} \Lambda$ final state [17], Panels c) and d) show the $p \pi^{+}$CMS angle of Reaction (7) (upper) and Reaction (8) (lower) [5]. Panel e) shows the $p \pi^{0}$ CMS angle of the exclusive $p p \pi^{0}$ final state [6]. While all the distributions refer to the data of $p+p$ collisions at a kinetic energy of $3.5 \mathrm{GeV}$ the last panel $\mathrm{f}$ ) shows data of $\mathrm{p}+\mathrm{p}$ collisions at the kinetic energy of $1.25 \mathrm{GeV}[8]$.

were reconstructed to raise the question whether resonances also play a key role in these channels. Figure 4 shows the answer to this question. The individual contributions of the single channels were fitted to the data [5]. The best fit result was achieved, with a dominant contribution of the case when the $\pi^{+} p$ stem from a $\Delta^{++}(1232)$ resonance instead of a direct production (11:1 for Reaction (7) and 7:1 for Reaction (8)). The non-resonant production of the $\pi^{+} p$ gave, in both cases, a negligible contribution to the mass spectra (red and brown histograms in Figure 4). Not only the ground state $\Delta$ resonance plays a significant role in strangeness production, but also heavier $\Delta$ resonances contribute to the kinematical properties of the measured particles. An example therefore is the $\Sigma(1385)^{+} K^{+}$ system of Reaction (3). We have investigated the $n-K^{+}$helicity angle (opening angle between $\Sigma(1385)$ and $\mathrm{n}$ in the $n-K^{+}$rest frame). This observable is suited to study the correlation of final state particles and is similar to an invariant mass [3]. The observable is displayed in Figure 5. While a nonresonant production of the $\Sigma(1385)^{+}$would yield an uniform distribution of the opening angle between the latter and the neutron, a production of the $\Sigma(1385)^{+}$as a decay product of a heavy $\Delta^{++}$resonance would lead to an anisotropic structure in this observable (blue histogram) [3]. A good description of the helicity distribution was achieved by including $1 / 3$ of the $\Sigma^{*}-K^{+}$pairs to be produced via a $\Delta^{++}$ with a mass of about $2035 \mathrm{MeV} / \mathrm{c}^{2}$. 

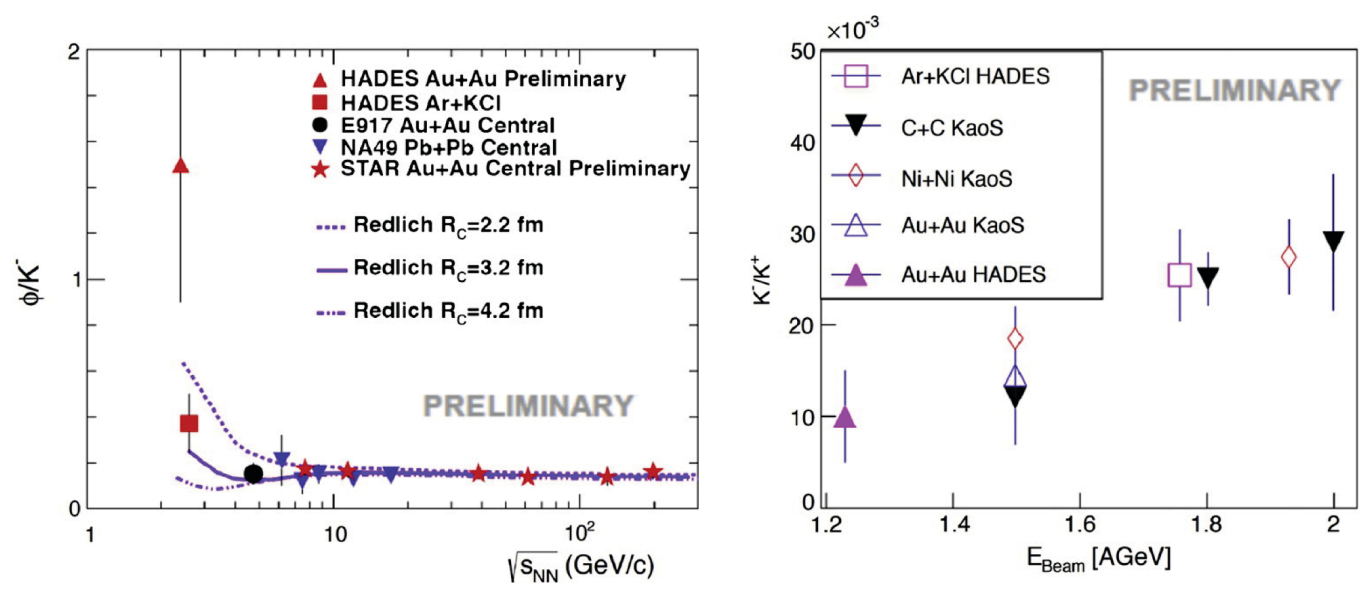

Figure 7. The left panel shows the preliminary $\phi / K^{-}$ratio at mid-rapidity measured by HADES in $\mathrm{Au}+\mathrm{Au}$ collisions at $1.23 \mathrm{GeV} / \mathrm{A}$ compared with published ratios [1]. The Au+Au value from HADES shows that the ratio strongly rises for low center of mass energies. This rise can be explained by a statistical model framework depending on the value of the strangeness correlation radius $R_{c}$ (violet curves). The right panel displays the $K^{-} / K^{+}$ratio as a function of the beam kinetic energy $[1,19]$. As the HADES data fit nicely into the general trend a certain trust is put in the reconstructed particle yields [20].

These examples show that already simple mass and helicity observables indicate that one can not neglect the presence of $\Delta$ resonances while modeling production processes, if one intends to describe the measured observables to a great detail.

\section{Angular Distributions}

Apart from the fact that HADES has shown in many ways how important $\Delta$ 's are as a source for hadrons, we have investigated the production kinematic of several exclusive final states. The observables of interest for that purpose are angular distributions in the center-of-mass reference frame of the $\mathrm{p}+\mathrm{p}$ colliding system. Figure 6 shows a compilation of several angular distributions of final states including strange and non-strange particles. The distributions are, in most cases, anisotropic with a preference for very forward and backward angles.

These observations show that a phase space production of particles without any underlying dynamics is not a good choice for a model to describe the data at SIS energies. This statement was in detail shown for the $p K^{+} \Lambda$ final state [17] for which the comparison to phase space simulation failed to describe the data [18]. Due to isospin conservation not $\Delta$ but $N^{*}$ resonances play the dominant role in this particular channel [7].

\section{Preliminary Results from the Au+Au System}

An analysis, currently performed on the $\mathrm{Au}(1.23 \mathrm{GeV} / \mathrm{A})+\mathrm{Au}$ data, shows once more the importance of understanding resonances as a source for light hadrons. In line with the findings from the previous $\operatorname{Ar}(1.76 \mathrm{GeV} / \mathrm{A})+\mathrm{KCl}$ run, where an essential result for strangeness physics was based in the determined $\phi / K^{-}$ratio of $0.37 \pm 0.13$ (meaning that around $20 \%$ of the $K^{-}$stem from the $\phi$ decay) [1], the new $\mathrm{Au}+\mathrm{Au}$ data hint towards an even more extreme role of the $\phi$ meson for the $K^{-}$properties. In the 
newly measured collision system about $70-80 \%$ of the $K^{-}$stem from the $\phi$ decay, making this resonance the dominant source for $K^{-}$[20]. Figure 7 shows the extracted $\phi / K^{-}$ratio with the preliminary systematic uncertainty. The ratio shows a strong rise towards low center of mass energies.

\section{Summary}

Here, many examples were shown to illustrate how important resonance properties are in order to understand the behavior of light hadrons. The idea of these diverse measurements performed by HADES is that the knowledge about particle production in elementary collisions should serve as an input to understand also heavier colliding systems. For that purpose, total and differential cross sections of exclusive final states have been reconstructed for strange and non-strange particle production. We have further investigated whether $\Delta$ resonances participate in the production of pions in the final state, which is mostly the case, especially for the $\Delta^{++}$resonance. Together with these results and the reconstruction of heavier strange resonances, the knowledge gained so far is a useful tool to understand the dynamics of heavy ion collisions with help of transport models.

\section{References}

[1] G. Agakishiev et al. [HADES Collaboration], Phys. Rev. C 80, 025209 (2009).

[2] M. Lorenz [HADES Collaboration], PoS BORMIO 2010, 038 (2010).

[3] G. Agakishiev et al. [HADES Collaboration], Phys. Rev. C 85, 035203 (2012).

[4] G. Agakishiev et al. [HADES Collaboration], Phys. Rev. C 87, 025201 (2013).

[5] G. Agakishiev et al. [HADES Collaboration], Phys. Rev. C 90, 015202 (2014).

[6] G. Agakishiev et al. [HADES Collaboration], Eur. Phys. J. A 50, 82 (2014).

[7] G. Agakishiev et al. [HADES Collaboration], Phys. Lett. B 742, 242 (2015)

[8] G. Agakishiev et al. [HADES Collaboration], Eur. Phys. J. A 48, 74 (2012).

[9] G. Agakishiev et al. [HADES Collaboration], Eur. Phys. J. A 41, 243 (2009).

[10] T. Hyodo and D. Jido, Prog. Part. Nucl. Phys. 67, 55 (2012).

[11] K. A. Olive et al. [Particle Data Group Collaboration], Chin. Phys. C 38, 090001 (2014).

[12] K. Moriya et al. [CLAS Collaboration], Phys. Rev. C 87, no. 3, 035206 (2013).

[13] J. Siebenson and L. Fabbietti, Phys. Rev. C 88, 055201 (2013).

[14] D. W. Thomas, A. Engler, H. E. Fisk and R. W. Kraemer, Nucl. Phys. B 56, 15 (1973).

[15] A. Engler, H. E. Fisk, R. w. Kraemer, C. M. Meltzer and J. B. Westgard, Phys. Rev. Lett. 15, 224 (1965).

[16] G. Agakishiev et al. [HADES Collaboration], Nucl. Phys. A 881, 178 (2012).

[17] E. Epple [HADES Collaboration], EPJ Web Conf. 81, 02005 (2014).

[18] L. Fabbietti et al. [HADES Collaboration], Nucl. Phys. A 914, 60 (2013).

[19] A. Forster et al., Phys. Rev. C 75, 024906 (2007).

[20] M. Lorenz [HADES Collaboration], Nucl. Phys. A 931, 785 (2014). 